\title{
Interstellar Abundances in the Magellanic Clouds
}

\author{
D. E. Welty, P. C. Frisch, L. M. Hobbs, and D. G. York \\ University of Chicago, 5640 S. Ellis Ave., Chicago, IL 60637, USA
}

J. C. Blades

STScI, 3700 San Martin Dr., Baltimore, MD 20771, USA

J. T. Lauroesch

Northwestern University, Evanston, IL 60208, USA

\section{G. Sonneborn}

Goddard Space Flight Center, Code 681, Greenbelt, MD 21218, USA

\begin{abstract}
We discuss the relative gas-phase abundances found for the predominantly neutral interstellar clouds - located in the Galactic disk and halo, in the LMC or SMC, and (perhaps) in between - along the lines of sight to Sk 108 in the SMC and to SN 1987A in the LMC.
\end{abstract}

In general, relative gas-phase interstellar abundances [e.g., $N(\mathrm{X} \mathrm{II}) / N(\mathrm{Zn}$ II)] reflect both the nucleosynthetic history of the gas and any selective depletion of elements into dust grains. HST GHRS echelle spectra (FWHM $\sim 4.2 \mathrm{~km} \mathrm{~s}^{-1}$ ) of Si II, Cr II, Mn II, Fe II, and Zn II reveal at least 25 interstellar absorption components toward Sk 108 (Welty et al. 1997). The relative abundances for the SMC components are similar to those found for clouds in the Galactic halo (Fig. 1, top). Toward SN 1987A, high-resolution (3-5 $\left.\mathrm{km} \mathrm{s}^{-1}\right)$ optical spectra of Ca II and Na I (Vidal-Madjar et al. 1987; Magain 1987) reveal at least 46 components, which may be associated with 10 component groups discernible in lower resolution (16-28 $\mathrm{km} \mathrm{s}^{-1}$ ) UV spectra of many species obtained with IUE. The relative abundances for the main LMC component groups toward the SN resemble those found for warm, diffuse Galactic disk clouds (Fig. 1, top); the relative abundances for the "intermediate velocity" groups are more similar to those found for the Galactic halo and SMC clouds; one group of Galactic halo clouds may be essentially dust-free (Welty et al. 1999). Since the stellar abundance ratios in the LMC and SMC are very similar those found locally in our Galaxy (for the elements we consider) (e.g., Russell \& Dopita 1992), we conclude that the depletion patterns are also similar in the three galaxies - despite differences in overall metallicity, dust-to-gas ratio, and UV extinction curves. Detailed studies of additional MC lines of sight, particularly with larger $E(B-V)$ and $N(\mathrm{H})$, are needed to confirm and extend that conclusion, however. Various diagnostic ratios suggest that the individual clouds in the various component groups toward SN 1987A are characterized by different physical conditions; most of the neutral 


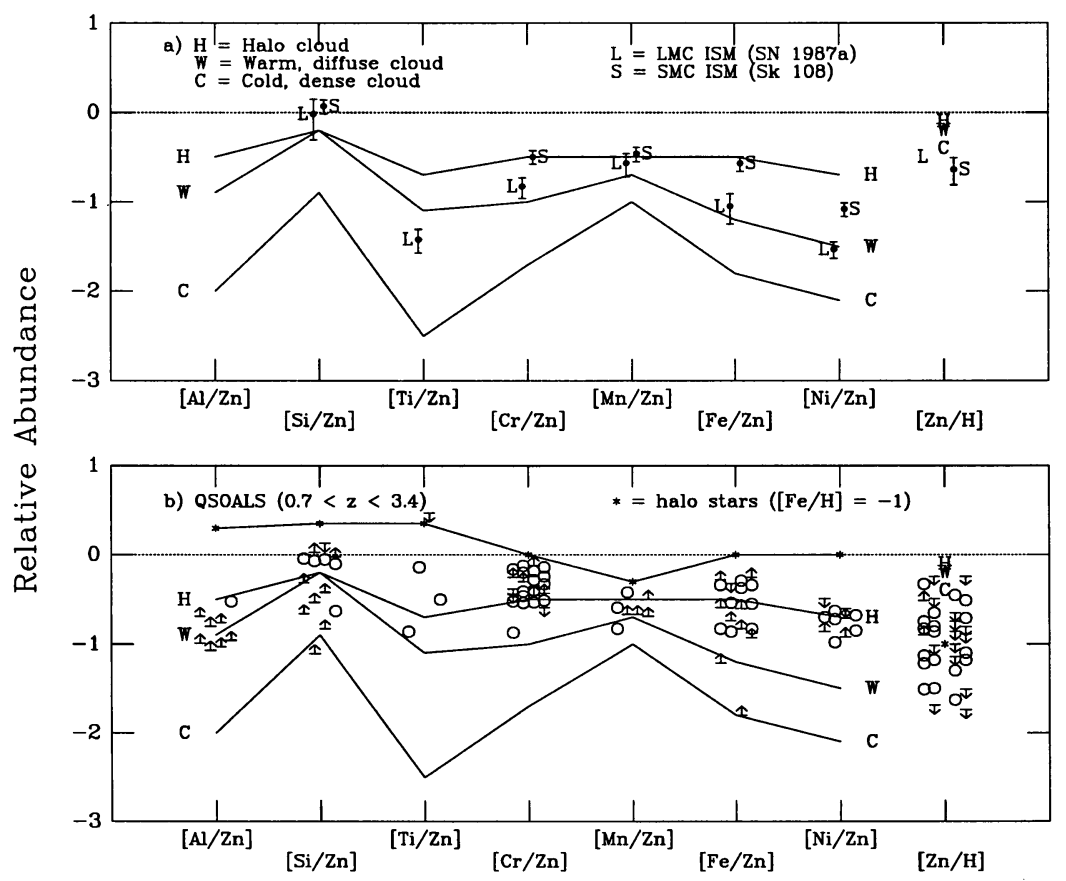

Figure 1. (top) Relative gas-phase abundances $\{[\mathrm{X} / \mathrm{Zn}]=\log [N(\mathrm{X}$ II $\left.) / N(\mathrm{Zn} \mathrm{II})]-\left(\mathrm{A}_{\mathrm{X}}-\mathrm{A}_{\mathrm{Zn}}\right)_{\odot}\right\}$ for main $\mathrm{SMC}$ gas toward $\mathrm{Sk} 108$ and main LMC gas toward SN 1987A, compared with patterns seen in Galactic cold, dense clouds (C), warm, diffuse clouds (W), and halo clouds $(\mathrm{H})$. (bottom) Relative gas-phase abundances seen for QSO absorption-line systems are similar, on average, to those in Galactic halo and SMC clouds. Individual systems exhibit a range of patterns, reflecting differences in nucleosynthetic history and/or depletions.

LMC gas has $T \lesssim 1500 \mathrm{~K}$, however. Determination of the relationships between gas, dust, and local physical conditions in the $\mathrm{MC}$ will aid in understanding the absorption-line systems seen toward distant QSO's, which also are characterized by low metallicities, low dust-to-gas ratios, mixtures of neutral and ionized components, and relative gas-phase abundances similar (on average) to those in the Galactic halo and SMC clouds (Fig. 1, bottom).

\section{References}

Magain, P. 1987, Nature, 329, 606

Russell, S. C., \& Dopita, M. A. 1992, ApJ, 384, 508

Vidal-Madjar, A., et al. 1987, A\&A, 177, L17

Welty, D. E., Frisch, P. C., Sonneborn, G., \& York, D. G. 1999, ApJ, 512, 636

Welty, D. E., Lauroesch, J. T., Blades, J. C., Hobbs, L. M., \& York, D. G. 1997, ApJ, 489, 672 